\title{
Telomere-related lung fibrosis is diagnostically heterogeneous but uniformly progressive
}

\author{
Chad A. Newton ${ }^{1,2}$, Kiran Batra ${ }^{3}$, Jose Torrealba ${ }^{4}$, Julia Kozlitina ${ }^{1}$, \\ Craig S. Glazer ${ }^{2}$, Carlos Aravena ${ }^{5}, K_{\text {Keith Meyer }}^{6}$, Ganesh Raghu $^{7}$, \\ Harold R. Collard ${ }^{5}$ and Christine Kim Garcia ${ }^{1,2}$
}

\begin{abstract}
Affiliations: ${ }^{1}$ Eugene McDermott Centre for Human Growth and Development, University of Texas Southwestern Medical Center at Dallas, Dallas, TX, USA. ${ }^{2}$ Dept of Medicine, University of Texas Southwestern Medical Center, Dallas, TX, USA. ${ }^{3}$ Dept of Radiology, University of Texas Southwestern Medical Center, Dallas, TX, USA. ${ }^{4}$ Dept of Pathology, University of Texas Southwestern Medical Center, Dallas, TX, USA. ${ }^{5}$ Dept of Medicine, University of California San Francisco, San Francisco, CA, USA. ${ }^{6}$ Dept of Medicine, University of Wisconsin, Madison, WI, USA. ${ }^{7}$ Dept of Medicine, University of Washington, Seattle, WA, USA.
\end{abstract}

Correspondence: Christine Kim Garcia, McDermott Centre; University of Texas Southwestern Medical Centre; 5323 Harry Hines Blvd.; Dallas, TX 75390-8591. E-mail: christine.garciadutsouthwestern.edu

ABSTRACT Heterozygous mutations in four telomere-related genes have been linked to pulmonary fibrosis, but little is known about similarities or differences of affected individuals.

115 patients with mutations in telomerase reverse transcriptase (TERT) (n=75), telomerase RNA component $(T E R C)(\mathrm{n}=7)$, regulator of telomere elongation helicase 1 (RTEL1) $(\mathrm{n}=14)$ and poly $(\mathrm{A})$ specific ribonuclease $(P A R N)(\mathrm{n}=19)$ were identified and clinical data were analysed.

Approximately one-half (46\%) had a multidisciplinary diagnosis of idiopathic pulmonary fibrosis (IPF); others had unclassifiable lung fibrosis (20\%), chronic hypersensitivity pneumonitis (12\%), pleuroparenchymal fibroelastosis (10\%), interstitial pneumonia with autoimmune features (7\%), an idiopathic interstitial pneumonia (4\%) and connective tissue disease-related interstitial fibrosis (3\%). Discordant interstitial lung disease diagnoses were found in affected individuals from $80 \%$ of families. Patients with TERC mutations were diagnosed at an earlier age than those with PARN mutations $(51 \pm 11$ years versus $64 \pm 8$ years; $\mathrm{p}=0.03)$ and had a higher incidence of haematological comorbidities. The mean rate of forced vital capacity decline was $300 \mathrm{~mL} \cdot \mathrm{year}^{-1}$ and the median time to death or transplant was 2.87 years. There was no significant difference in time to death or transplant for patients across gene mutation groups or for patients with a diagnosis of IPF versus a non-IPF diagnosis.

Genetic mutations in telomere related genes lead to a variety of interstitial lung disease (ILD) diagnoses that are universally progressive.

@ERSpublications

Mutations in four telomere-related genes lead to progressive pulmonary fibrosis, regardless of ILD diagnosis http://ow.ly/TH2B300Yjvt

Editorial comment in: Eur Respir J 2016; 48: 1556-1558.

This article has supplementary material available from erj.ersjournals.com

Received: Feb 102016 | Accepted after revision: May 312016 | First published online: Aug 182016

Support statement: The authors acknowledge funding support from the following sources: US National Institutes of Health, US National Heart, Lung, and Blood Institute: 5T32HL098040 (C.A. Newton), UL1TR001105 and R01HL093096 (C.K. Garcia); and the National Centre for Advancing Translational Science. Funding information for this article has been deposited with the Open Funder Registry.

Conflict of interest: Disclosures can be found alongside this article at erj.ersjournals.com

Copyright OERS 2016 


\section{Introduction}

The interstitial lung diseases are a heterogeneous collection of diseases characterised by deposition of extracellular matrix within the pulmonary interstitium leading to cough, shortness of breath and hypoxia. The prototype, idiopathic pulmonary fibrosis (IPF), is a distinct type of chronic lung fibrosis that demonstrates a devastating progressive decline in lung function usually resulting in respiratory failure $[1,2]$. The cause of IPF remains unknown, but recent investigations have implicated telomere shortening as a key contributor to its pathogenesis and survival characteristics [3-5].

Telomerase is a reverse transcriptase enzyme that adds TTAGGG repeats to chromosomal ends during cell replication. Mutations in the protein component of telomerase (TERT) and the RNA component of the enzyme (TERC) lead to short telomere lengths and familial pulmonary fibrosis $[6,7]$. TERT mutations are the most common mutation found in individuals with familial pulmonary fibrosis as they are found in $\sim 15 \%$ of affected kindreds [8]. Mutations in dyskerin (DKC1) and TRF1-interacting protein 2 (TINF2) have also been found in patients with familial interstitial pneumonia $[9,10]$. Recently, two other genes, RTEL1 and PARN, have been associated with shortened telomere lengths and familial pulmonary fibrosis [11-13]; thus, six telomere-related genes have been linked to this disease. RTEL1 encodes for protein that contains an N-terminal helicase that unwinds the telomere T-loop structures during DNA replication. PARN contributes to the poly (A)-specific maturation of TERC RNA and maintenance of telomere length [14]. Evidence for a gene dosage exists as two copies of deleterious TERT [15], RTEL1 [16-18] and PARN [14, 19, 20] mutations have been found in young children with dyskeratosis congenita and multiorgan disease that includes pulmonary fibrosis.

Clinically, a diagnosis of IPF predicts more rapid disease progression and a shorter life expectancy compared with the non-IPF interstitial lung diseases [21]. Telomere length has also been shown to be associated with a shorter life expectancy in IPF [5, 22] and prior descriptions of pulmonary fibrosis patients with TERT mutations have shown that many have a clinical diagnosis of IPF [6-8]. We sought to test the hypothesis that patients with pulmonary fibrosis due to heterozygous genetic mutations in TERT, TERC, RTEL1 or PARN share a common clinical phenotype (IPF) and a progressive clinical course.

\section{Methods}

Study design and participants

In this observational cohort study, patients with familial pulmonary fibrosis were identified at the University of Texas Southwestern Medical Centre (UTSW; Dallas, TX, USA) or were referred from different academic medical centres for participation. This study was approved by the UTSW institutional review board and written informed consent was obtained from all living subjects. 38 (59\%) of 64 kindreds were previously reported; clinical data of each case has been independently reviewed in this study. For 28 cases, the presence of a genetic mutation in an affected individual could be inferred from pedigree structure and genotyping of living family members. In all these cases, the presence of a mutation is imputed only for individuals who are obligate carriers based upon their position in the pedigree between subjects with confirmed variants. Clinical information on subjects including demographics, laboratory results, pulmonary function testing, radiographic studies and pulmonary histopathological specimens were collected. Ethnicity was self-reported or family-reported. Genomic DNA samples from healthy control participants aged 19-89 years [3, 5, 8] were obtained from a cohort of unrelated, multiethnic individuals from Dallas, TX, USA.

\section{Procedures}

Genomic DNA was isolated from circulating leukocytes using an Autopure LS (Qiagen, Valencia, CA, USA). Telomere lengths of genomic DNA from circulating leukocytes were measured using a quantitative PCR assay as previously described $[3,5,8]$ and are represented as a logarithm-transformed relative ratio of telomeres to a single copy gene (T/S) or an age-adjusted telomere length. Sequencing the TERT, TERC, RTEL1 and PARN genes was performed as previously described $[7,11]$. No mutations in DKC1 [9] or TINF2 [10] were found in this cohort. Clinical workup and management was directed by local physicians. To account for variation in practices, all data were independently reviewed using current clinical guidelines to establish a multidisciplinary diagnosis $[2,23,24]$.

Available chest computed tomography (CT) images $(n=73)$ were reviewed by a chest radiologist (K. Batra) who was blinded to clinical history, mutation analysis, telomere length and histopathology. The scans were graded for 31 distinct radiographic features and were classified into one of three radiographic patterns: definite usual interstitial pneumonia (UIP), possible UIP and inconsistent with UIP [2].

Available lung histopathological specimens $(n=42)$ were analysed by a pulmonary pathologist ( $J$. Torrealba) who was blinded to clinical history, mutation analysis, telomere length and radiographic characteristics. Available specimens, including surgical lung biopsies and lung explants, were graded for 15 different histopathological features. Each specimen was given a histopathologic diagnosis according to established guidelines [23]. 


\section{Statistical analysis}

Characteristics of patient groups were compared using one-way ANOVA (for continuous variables) or Fisher's exact test (for categorical variables). The differences in the age at diagnosis were tested using linear regression, including gene and generation as predictors (or by a t-test, when there were only two generations). Telomere length relative T/S ratios were logarithm-transformed to ensure that residuals were normally distributed and had constant variance. We estimated the relation between telomere length and age using linear regression of control participants, as previously described [5]. The estimated regression coefficients were used to calculate the observed minus expected, or age-adjusted, telomere length of each subject.

Changes in pulmonary function test (PFT) characteristics were analysed using linear mixed-effects models for patients with at least two separate PFT measurements. We included age and sex as covariates to account for differences in age and the time of the first PFT measurement and in sex proportions for the various genes. To test whether the rate of change in PFT depended on age and sex, we initially included the agextime and sex $\times$ time interaction terms in the model. In separate models, we tested whether the average PFT characteristics or the rate of change in PFT depended on telomere length or diagnosis, by including the terms for the fixed effects of telomere length ( $>10$ th percentile or $<10$ th percentile) or diagnosis (IPF or non-IPF) and time-dependent interaction terms in the model. Terms that were not statistically significant ( $p$-value $>0.05$ ) were excluded from the final model. The parameters were estimated using the restricted maximum likelihood procedure. The need for the random effects was assessed using likelihood ratio tests.

Median transplant-free survival time (time from diagnosis to transplant or death) and overall survival was calculated using the Kaplan-Meier curves. Cox proportional hazards models were used to assess the effect of age, sex, clinical parameters (forced vital capacity (FVC) \% predicted and diffusing capacity of the lung for carbon monoxide (DLCO) \% predicted), gene mutation carrier group, telomere length, and diagnosis (IPF versus non-IPF) on transplant-free survival time. All analyses were performed using $\mathrm{R}$ version 3.2.2 statistical analysis software (www.R-project.org).

\section{Results}

\section{Patient characteristics}

We identified 115 individuals with pulmonary fibrosis from 64 families with mutations in one of four genes linked to telomere shortening: TERT, TERC, RTEL1 and PARN ( $\mathrm{n}=75,7,14$ and 19, respectively). Each genetic mutation was rare and unique to the family in which it was discovered. For 38 (60\%) families only one affected individual was included (supplemental table S1). Characteristics of subjects with monogenic pulmonary fibrosis are described in tables 1 and 2. Most affected individuals were Caucasian. More affected females were found with TERC (86\%) or PARN (53\%) mutations, although this result did not reach statistical significance in the comparison across all gene groups. While the mean age at diagnosis for all mutation carriers was 58 years old, individuals with TERC mutations were diagnosed at a younger mean age (51 \pm 11 years) and $P A R N$ mutation carriers were diagnosed at a later mean age (64 \pm 8 years, $\mathrm{p}=0.03$ ). We find no evidence for an effect of smoking on the age of diagnosis. Compared with subjects with TERT mutations, the age-adjusted telomere lengths of the PARN mutation carriers were longer $(-0.36 \pm 0.14$ versus $-0.58 \pm 0.27 ; \mathrm{p}=0.013)$. Similarly, a higher percentage of $P A R N$ mutation carriers had blood leukocyte telomere length $>10$ th percentile than the entire cohort ( $40 \%$ versus $13 \%$; $\mathrm{p}=0.049$ ).

Anaemia and macrocytosis were the most common haematological abnormalities, found in $28 \%$ and $24 \%$ of the total cohort, respectively. TERC mutation carriers with pulmonary fibrosis had a higher incidence of leukopenia, thrombocytopenia, aplastic anaemia or myelodysplastic syndrome $(\mathrm{p}<0.05)$ (table 2$)$. Two subjects with RTEL1 mutations (14\%) had lung cancer, which is statistically significant when compared with the other groups $(\mathrm{p}=0.017)$. The overall incidence of cancer was $9.7 \%$. Additional comordid conditions are presented in supplemental table S2.

\section{Clinical ILD diagnoses}

Of the 77 subjects for whom there was sufficient data to make a multidisciplinary diagnosis, 35 (46\%) had a diagnosis of IPF (table 1). The most common non-IPF diagnoses included unclassifiable lung fibrosis (20\%), chronic hypersensitivity pneumonitis $(12 \%)$ and pleuroparenchymal fibroelastosis (10\%) (figure 1a-f). Two patients with connective tissue disease-associated ILD had a rheumatological diagnosis of scleroderma. There was no statistically significant difference in the distribution of ILD diagnoses across gene groups.

Affected members of the same family often have discordant diagnoses, despite their inheritance of the same germline genetic mutation. Of the 15 different families for whom a multidisciplinary ILD diagnosis was made for $\geqslant 2$ affected family members (table 3 ), a discordant diagnosis was seen across affected individuals in $12(80 \%)$ families and a concordant diagnosis of IPF was found for three (20\%) families. Figure 1i-l displays lung histopathology of individuals with discordant diagnoses from two families. 
TABLE 1 Characteristics of interstitial lung disease subjects with heterozygous TERT, TERC, RTEL1 or PARN mutations

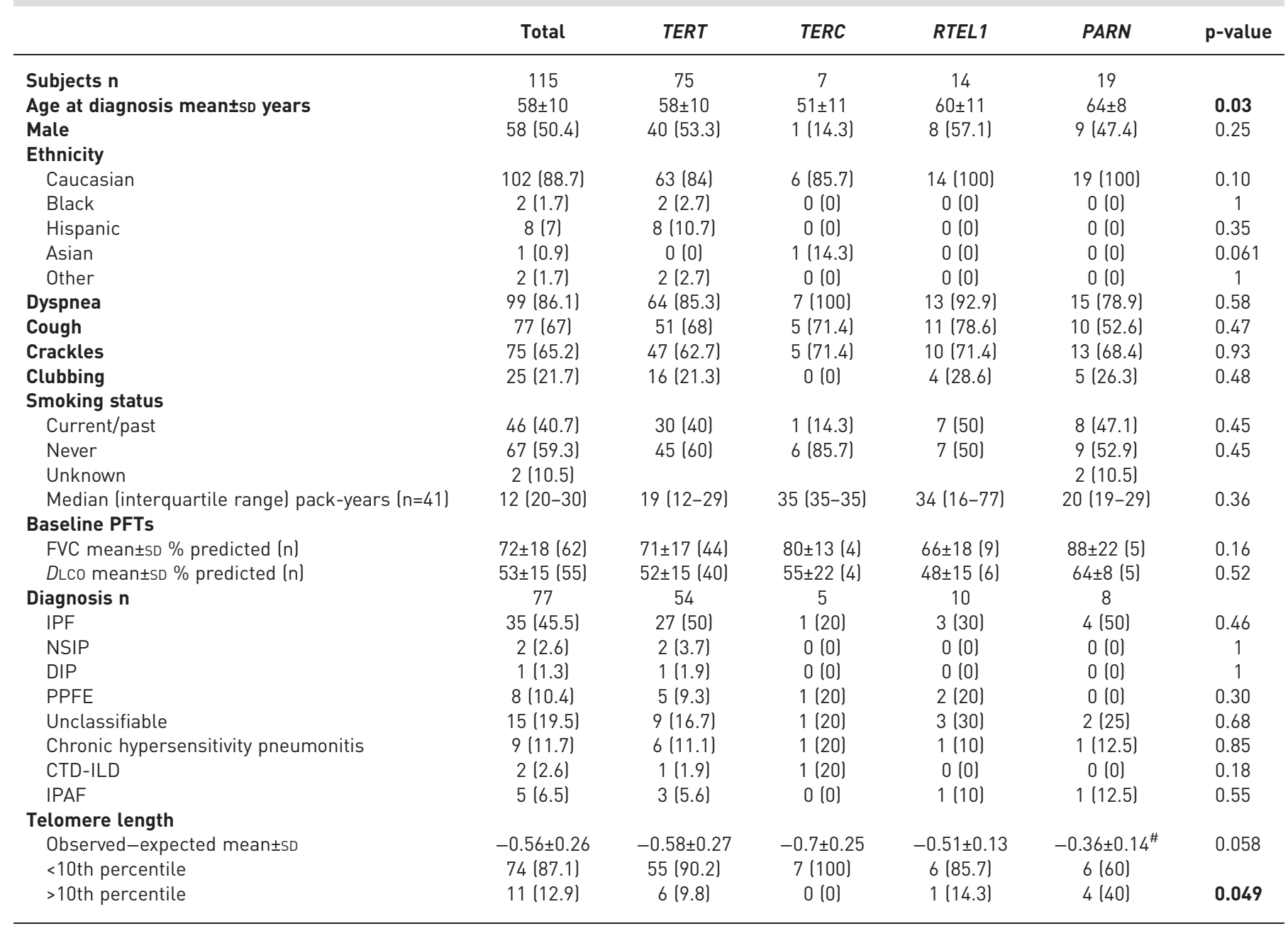

Data are presented as $\mathrm{n}(\%)$, unless otherwise stated. ${ }^{\text {: }}$ different from TERT group with a $\mathrm{p}$-value of 0.013 . $\mathrm{p}$-values $<0.05$ are shown in bold. PFTs: pulmonary function tests; FVC: forced vital capacity; DLCO: diffusion capacity of the lung for carbon monoxide; IPF: idiopathic pulmonary fibrosis; NSIP: nonspecific interstitial pneumonia; DIP: desquamative interstitial pneumonia; PPFE: pleuroparenchymal fibroelastosis; CTD-ILD: connective tissue disease-associated interstitial lung disease; IPAF: idiopathic pneumonia with autoimmune features.

\section{Chest CT and histopathological correlations}

73 cases had chest CT scans available for review. These were classified into one of three radiographic patterns: definite UIP, possible UIP and inconsistent with UIP (supplemental table S3). Nearly one-half (47\%) of all CT scans represented a definite UIP pattern. Overall, one-third of the scans were consistent with possible UIP and $20 \%$ of the scans were found to be inconsistent with a UIP pattern.

We found a significant association between emphysema and gene group $(p=0.032)$. Compared with other gene groups, a higher proportion of RTEL1 mutation carriers (40\%) and TERT mutation carriers (40\%) had radiographic evidence of emphysema ( $p=0.026$ and $p=0.028$, respectively; supplemental table $S 4)$. We also found an association between emphysema and smoking $(\mathrm{p}=0.015)$.

42 surgical lung specimens were available for review (supplemental table S5). A histopathological diagnosis of UIP was found in 21 (50\%) of subjects. Microscopic honeycombing was found in $33(78 \%)$ subjects and fibroblastic foci were seen in $34(80.9 \%)$ subjects. There was no statistically significant difference in histopathological patterns across subjects with different genetic mutations.

We assessed the correlation between the radiographic and histopathological diagnoses of 34 subjects for whom both chest CT and histopathologic specimens were available. A pathologic diagnosis of UIP was seen in $61 \%$ and $38 \%$ of cases with a radiographic diagnosis of definite UIP and possible UIP, respectively (supplemental table S6). One-half of cases with a chest CT scan that was found to be inconsistent with UIP had a histopathological diagnosis of UIP. 


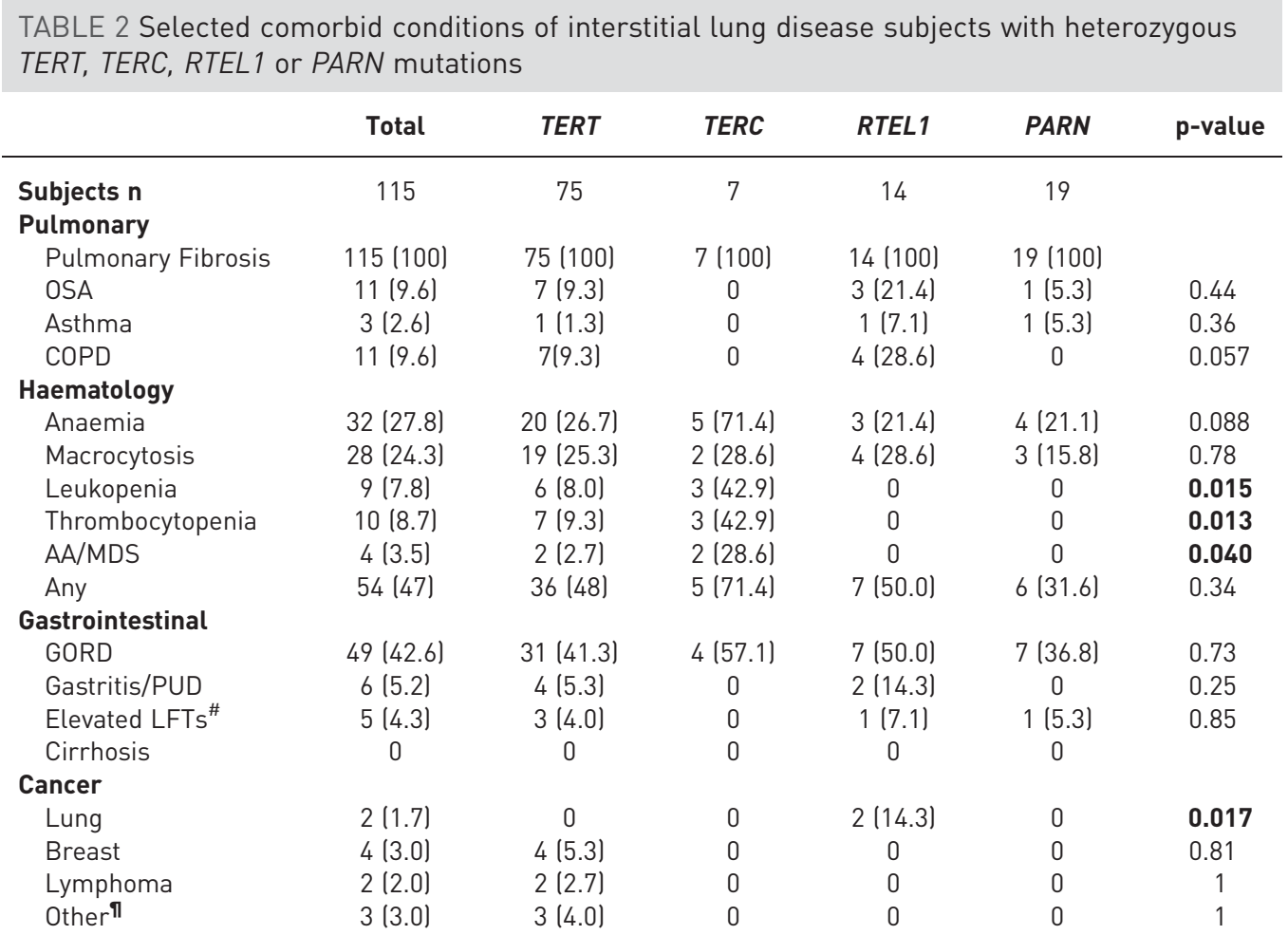

Data are presented as $\mathrm{n}(\%)$, unless otherwise stated. The prevalence of comorbid conditions is compared using Fisher's exact test. p-values <0.05 are shown in bold. OSA: obstructive sleep apnoea; COPD: chronic obstructive pulmonary disease; AA: aplastic anaemia; MDS, myelodysplastic syndrome; GORD: gastro-oesophageal reflux disease; PUD: peptic ulcer disease; LFT: liver function tests. \#: LFTs were transiently elevated in three of the five individuals; it was attributed to a drug (dapsone)-induced liver injury in one RTEL1 mutation carrier. The aetiology of the transient elevation was unknown for the other cases. The two TERT mutation carriers with a persistent elevation of LFTs were infected with hepatitis $\mathrm{C}$ and both received interferon/ribavirin therapy. All five of these individuals had abdominal imaging and none were found to have cirrhosis. ": non-melanoma skin cancers were excluded. Others include oral squamous cell, endometrial and ovarian cancer.

\section{Genetic anticipation}

In this cohort, there were nine families (four with TERT, two with RTEL1 and three with PARN mutations) with individuals from more than one generation. We found evidence for genetic anticipation in carriers of TERT and RTEL1 mutations ( $\mathrm{p}=0.003$ and $\mathrm{p}=0.018$, respectively), but not PARN mutations (figure $2 \mathrm{a}$ ).

\section{Disease progression and survival}

The mean decline in FVC was $300 \mathrm{~mL} \cdot \mathrm{year}^{-1}$ (range 260-350 $\mathrm{mL} \cdot \mathrm{year}^{-1}$ across gene groups) (table 4). The mean decline in DLCO was $1.7 \mathrm{~mL} \cdot \mathrm{min}^{-1}$ per $\mathrm{mmHg} \cdot \mathrm{year}^{-1}$. There was no significant difference in the rate of FVC or DLCO decline across genes or across diagnoses. Notably, the mean rate of decline in FVC was $299 \mathrm{~mL} \cdot$ year $^{-1}$ and $296 \mathrm{~mL} \cdot$ year $^{-1}$ for those with a diagnosis of IPF or a non-IPF diagnosis, respectively.

59 (51\%) patients died and 34 (30\%) underwent lung transplantation over the follow-up period (table 5). Overall, the median time to death or transplantation was 2.87 years (95\% CI 2.40-3.80) for all patients. The median time to death or transplantation is not significantly different across gene groups or between those with IPF or a non-IPF diagnosis. The transplant-free survival of ILD patients across the four gene groups was not significantly different $(p=0.63)$ and is shown in figure $2 b$. Overall survival was also not significantly different across the four groups ( $\mathrm{p}=0.86$, data not shown). The transplant-free survival of patients with a mutation in one of these four genes was not significantly different for those with a diagnosis of IPF or a non-IPF diagnosis $(\mathrm{p}=0.25)$ and is shown in figure $2 \mathrm{c}$.

In an unadjusted Cox analysis, baseline FVC \% predicted was strongly associated with transplant-free survival for patients (HR 0.65 (95\% CI 0.53-0.79) for each 10 percentage point FVC difference, p<0.0001) (table 6). Other unadjusted predictors of transplant-free survival included age at the time of diagnosis (HR 1.02 (1-1.04), $\mathrm{p}=0.046)$ and male sex (HR $1.6(1.04-2.44), \mathrm{p}=0.031)$. The various gene mutations, telomere length and 


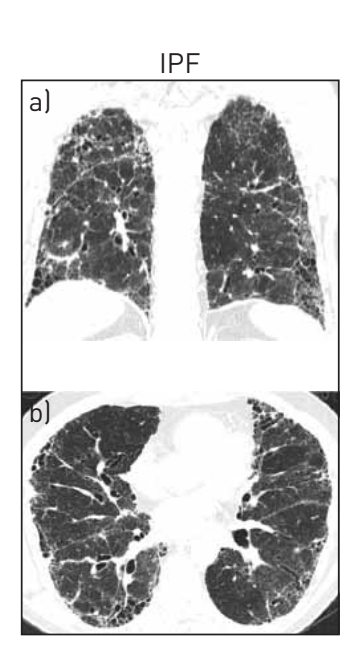

Chronic hypersensitivity pneumonitis
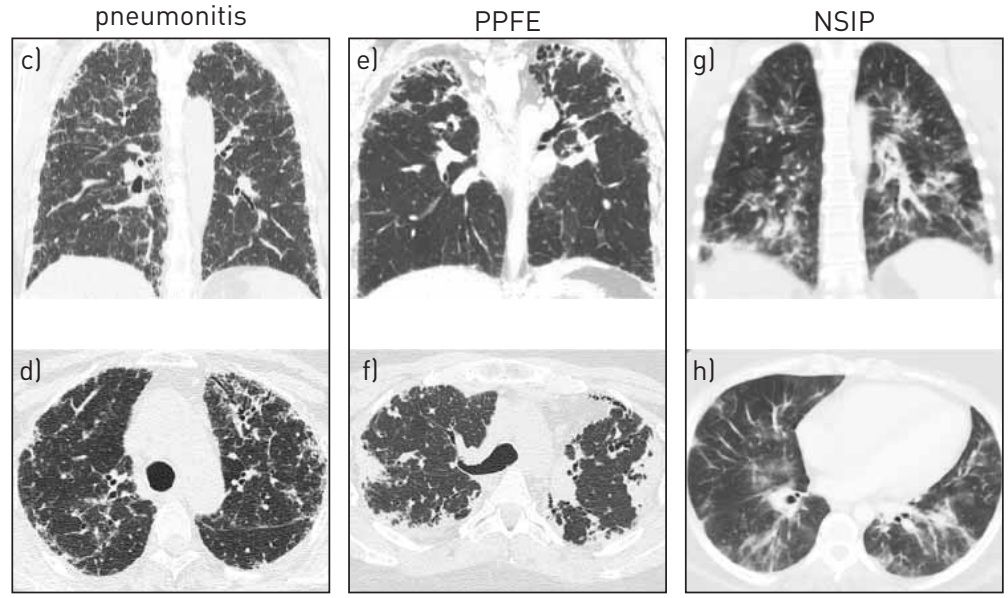

UIP
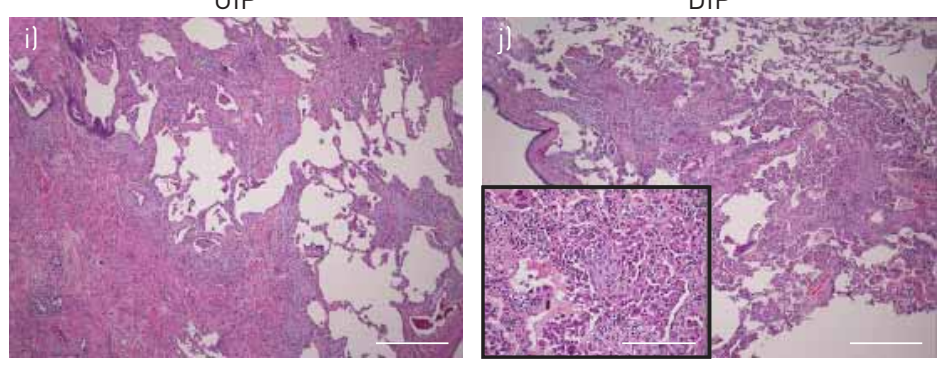

Chronic hypersensitivity

PPFE pneumonitis

amily members with same TERT mutation
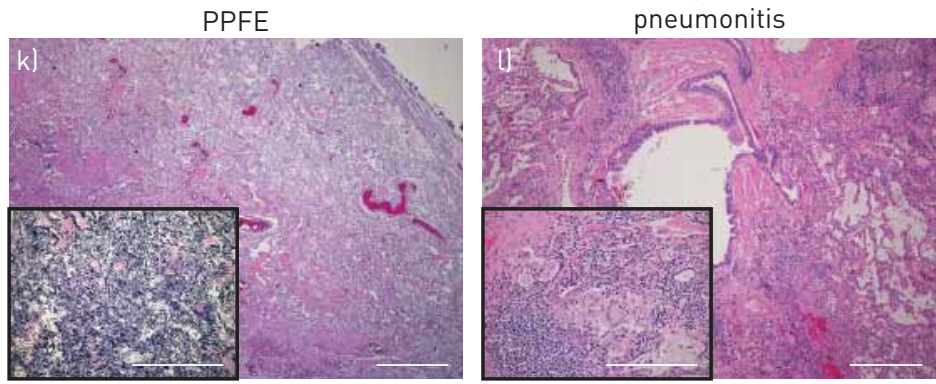

FIGURE 1 Spectrum of interstitial lung disease diagnoses in subjects with heterozygous TERT, TERC, RTEL1 or PARN mutations. Representative coronal $(a, c, e, g)$ and axial $(b, d, f, h)$ images of chest computed tomography scans from patients with diagnoses of $a$ and b) idiopathic pulmonary fibrosis (IPF), $c$ and d) chronic hypersensitivity pneumonitis, e and f) pleuroparenchymal fibroelastosis (PPFE) and $g$ and $h$ ) nonspecific interstitial pneumonia (NSIP). Representative haematoxylin and eosin stained lung sections from related ILD subjects with $i$ and $j$ ) the same TERT c.2594G>A (R865H) mutation and $\mathrm{k}$ and () the same TERC r.182g >c mutation. i) Usual interstitial pneumonia (UIP) pattern with temporal and spatial heterogeneity and fibroblastic foci at the interface between normal and fibrotic lung in a 53-year-old man who underwent lung transplantation. j) Desquamative interstitial pneumonia (DIP) with organising pneumonia, thickened interstitial septi and presence of intra-alveolar pigmented macrophages (inset) in a 68-year-old never-smoker (scale bar $=500 \mu \mathrm{m}$; inset scale bar=200 $\mu \mathrm{m}$ ). k) Pleuroparenchymal fibroelastosis (PPFE) with diffuse elastic fibre deposition (inset) near the pleural edge in a lung explant from a 60-year-old female at the time of lung transplantation (scale bar $=500 \mu \mathrm{m}$; inset scale bar=500 $\mu \mathrm{m}$ ). () Features of chronic hypersensitivity pneumonitis with bronchiolocentric fibrosis, patchy lymphoid aggregates, bridging fibrosis and loosely formed granulomas (inset) in a lung explant from her 57 -year-old sister (scale bar=500 $\mu \mathrm{m}$; inset scale bar=500 $\mu \mathrm{m}$ ).

diagnosis subtype (IPF versus non-IPF) were not significantly associated with survival in unadjusted analysis. After adjustment for relevant individual covariates, FVC \% predicted remained an independent predictor of transplant-free survival time (HR $0.93(0.89-0.98), \mathrm{p}=0.004)$ but telomere length did not.

\section{Discussion}

Genetic mutations in TERT, TERC, RTEL1 and PARN are associated with development of a short telomere syndrome, or "telomeropathy" that can be characterised by systemic abnormalities including pulmonary 
TABLE 3 Multidisciplinary diagnoses of interstitial lung disease (ILD) subjects with heterozygous TERT, TERC, RTEL1 or PARN mutations from the same kindred

\begin{tabular}{|c|c|c|}
\hline Gene & Mutation & Multidisciplinary diagnoses of family members with ILD \\
\hline \multicolumn{3}{|c|}{ Concordant } \\
\hline TERT & p.Thr874Arg & IPF, IPF \\
\hline TERT & p.His925Gln & IPF, IPF \\
\hline TERT & p.Gly1063Ser & IPF, IPF \\
\hline \multicolumn{3}{|c|}{ Discordant } \\
\hline TERT & p.Val144Met & PPFE, chronic hypersensitivity pneumonitis, unclassifiable \\
\hline TERT & p.Arg486Cys & IPF, IPAF \\
\hline TERT & p.Arg631Gln & IPF, PPFE \\
\hline TERT & p.Pro702Leu & IPF, IPF, unclassifiable \\
\hline TERT & p.Arg $865 \mathrm{His}$ & IPF, IPF, IPF, DIP, unclassifiable \\
\hline TERT & p.Arg951Trp & PPFE, IPF \\
\hline TERT & p.Leu1019Phe & Chronic hypersensitivity pneumonitis, IPAF \\
\hline TERC & $r .182 g>c$ & Chronic hypersensitivity pneumonitis, PPFE \\
\hline RTEL 1 & p.Gly201GlufsX15 & IPF, IPAF \\
\hline RTEL 1 & p.Pro484Leu & Unclassifiable, IPF \\
\hline RTEL 1 & p.Gln669X & Chronic hypersensitivity pneumonitis, PPFE \\
\hline PARN & c. $246-2 A>G$ & Unclassifiable, IPAF \\
\hline
\end{tabular}

IPF: idiopathic pulmonary fibrosis; IPAF: interstitial pneumonia with autoimmune features; PPFE: pleuroparenchymal fibroelastosis; DIP: desquamative interstitial pneumonia.

fibrosis, bone marrow dysfunction, liver cirrhosis and early greying. Until now, phenotypic descriptions of individuals with inherited mutations in these genes have been limited to reports of paediatric patients with dyskeratosis congenita and adult patients with various manifestations of this disease spectrum. Overall, pulmonary fibrosis appears to be the most common phenotype associated with telomerase mutations [25]. Here, we studied a large collection of older adult patients with rare heterozygous telomere-related mutations who were collected based upon the familial pulmonary fibrosis phenotype. We found that this cohort exhibited similarities and differences in the clinical expressivity of haematological, gastrointestinal and emphysema phenotypes that have been noted in other cohorts [26-28]. For example, we found that severe haematological diseases were found more often in those with TERC mutations. The hepatic

TABLE 4 Forced vital capacity and diffusion capacity change over time for interstitial lung disease subjects with heterozygous TERT, TERC, RTEL 1 or PARN mutations

\begin{tabular}{|c|c|c|c|c|c|c|}
\hline PFT parameter & Group & $\mathbf{N}$ & Mean number of measurements & Mean follow-up time years & Mean change per year & p-value \\
\hline \multirow{3}{*}{ FVC L } & TERT & 46 & 3.76 & 2.16 & -0.30 & NS \\
\hline & TERC & 6 & 4.67 & 2.23 & -0.26 & \\
\hline & PARN & 9 & 4.22 & 3.30 & -0.27 & \\
\hline \multirow[t]{3}{*}{ FVC \% predicted } & All & 71 & 3.83 & 2.40 & -7.40 & \\
\hline & TERT & 46 & 3.67 & 2.13 & -7.48 & NS \\
\hline & PARN & 9 & 4.22 & 3.30 & -7.87 & \\
\hline \multirow[t]{5}{*}{$D$ Lco $\mathrm{mL} \cdot \mathrm{min}^{-1} \cdot \mathrm{mmHg}^{-1}$} & All & 67 & 3.39 & 2.30 & -1.73 & \\
\hline & TERT & 44 & 3.25 & 2.04 & -1.73 & NS \\
\hline & TERC & 5 & 4.00 & 2.26 & -0.81 & \\
\hline & RTEL 1 & 9 & 3.11 & 2.62 & -2.11 & \\
\hline & PARN & 9 & 4.00 & 3.30 & -1.80 & \\
\hline DLco \% predicted & PARN & 9 & 4.11 & 3.30 & -7.53 & \\
\hline
\end{tabular}

NS: nonsignificant; PFT: pulmonary function test; FVC: forced vital capacity; DLCo: diffusion capacity of the lung for carbon monoxide. 
TABLE 5 Cumulative events and time to death or lung transplantation for interstitial lung disease subjects with TERT, TERC, RTEL1 or PARN mutations

\begin{tabular}{|c|c|c|c|c|c|c|c|}
\hline & \multirow[t]{2}{*}{ Subjects $\mathrm{n}$} & \multirow[t]{2}{*}{ Death } & \multirow[t]{2}{*}{ Transplant } & \multirow[t]{2}{*}{ Death or transplant } & \multicolumn{3}{|c|}{ Time to death or transplant years } \\
\hline & & & & & $\mathbf{n}$ & Events $\mathrm{n}$ & Median $(95 \% \mathrm{CI})$ \\
\hline Total & 115 & $59(51)$ & $34(30)$ & $93(81)$ & 106 & 88 & $2.87(2.40-3.80)$ \\
\hline TERC & 7 & $3(43)$ & 2 (29) & $5(71)$ & 7 & 5 & $2.54(1.92-\mathrm{NA})$ \\
\hline RTEL1 & 14 & $8(57)$ & $2(14)$ & $10(71)$ & 13 & 9 & $2.87(2.30-\mathrm{NA})$ \\
\hline PARN & 19 & $12(63)$ & $3(16)$ & $15(79)$ & 16 & 13 & $5.73(2.55-\mathrm{NA})$ \\
\hline Non-IPF & 42 & $18(43)$ & $15(36)$ & $33(79)$ & 42 & 33 & $3.11(2.56-4.82)$ \\
\hline
\end{tabular}

Data are presented as n (\%), unless otherwise stated. IPF: idiopathic pulmonary fibrosis.

phenotype we observed in this cohort appears to be milder than has been described by others [27, 29]. And the radiographic emphysema phenotype was not only significantly associated with smoking and the TERT mutation group as has been recently described [28], but also associated with the RTEL1 gene.

Of the 77 subjects in this cohort for whom a multidisciplinary diagnosis was made, we found that $46 \%$ met diagnostic criteria for IPF. The other diagnoses included other idiopathic interstitial pneumonias of unknown cause (nonspecific interstitial pneumonia, desquamative interstitial pneumonia, pleuroparenchymal fibroelastosis) as well as ILD attributed to known causes (chronic hypersensitivity pneumonitis and connective tissue disease-associated ILD). Over 50\% of affected individuals had a diagnosis other than IPF. Other studies of ILD patients with short telomere lengths have also found high percentages of non-IPF diagnoses, including hypersensitivity pneumonitis, unclassified interstitial pneumonia and combined pulmonary fibrosis and emphysema [30, 31]. We found discordant diagnoses among affected individuals with the same inherited rare mutation in $80 \%$ of kindreds. This degree of phenotypic heterogeneity in the same family was higher than originally found by STEELE et al. [32] in a familial interstitial pneumonia cohort (45\%).

There is poor genotype-ILD phenotype correlation across patients with different telomere-related genetic mutations. The location of the lung fibrosis (lower lobe, upper lobe, peripheral, airway-centric), the pattern of lung destruction (microcystic versus macrocystic honeycombing), the nature of extracellular matrix deposition in the lung (collagen, elastin, homogeneity of haematoxylin-stained proteins) and the temporal extent of remodelling (homogeneous versus heterogeneous) are likely to be controlled by other factors, both genetic and environmental. For example, the common MUC5B risk polymorphism (rs35705950) is associated with a radiographic and histopathological UIP pattern [33]. Sex may be another contributing

TABLE 6 Analysis of transplant-free survival for interstitial lung disease subjects with TERT, TERC, RTEL1 or PARN mutations

\begin{tabular}{|c|c|c|c|c|c|}
\hline & \multirow[t]{2}{*}{$\mathbf{N}$} & \multicolumn{2}{|c|}{ Univariable analysis } & \multicolumn{2}{|c|}{ Multivariable analysis ( $n=36$ ) } \\
\hline & & Hazard ratio $(95 \% \mathrm{CI})$ & p-value & Hazard ratio $(95 \% \mathrm{CI})$ & p-value \\
\hline Age at diagnosis & 106 & $1.02(1-1.04)$ & 0.048 & $1.05(0.99-1.12)$ & 0.096 \\
\hline Male & 106 & $1.6(1.04-2.44)$ & 0.031 & $0.95(0.28-3.23)$ & 0.93 \\
\hline FVC $\%$ predicted ${ }^{\#, \eta}$ & 62 & $0.65(0.53-0.79)$ & $<0.0001$ & $0.49(0.3-0.79)$ & 0.0035 \\
\hline DLco \% predicted $\#$, пा & 55 & $0.81(0.64-1.02)$ & 0.071 & $1.32(0.79-2.21)$ & 0.28 \\
\hline \multicolumn{6}{|l|}{ Gene } \\
\hline TERT & & Ref & & Ref & \\
\hline TERC & 106 & $0.79(0.32-1.98)$ & 0.61 & $0.67(0.06-7.56)$ & 0.74 \\
\hline RTEL1 & 106 & $0.75(0.37-1.51)$ & 0.42 & $0.58(0.11-2.96)$ & 0.51 \\
\hline PARN & 106 & $0.72(0.39-1.31)$ & 0.28 & $0.26(0.06-1.19)$ & 0.083 \\
\hline Log (T/S) telomere length & 80 & $0.76(0.3-1.93)$ & 0.57 & $6.18(0.91-42.13)$ & 0.063 \\
\hline IPF versus non-IPF & 77 & $1.34(0.81-2.23)$ & 0.25 & $0.46(0.18-1.22)$ & 0.12 \\
\hline
\end{tabular}

FVC: forced vital capacity; DLCO: diffusion capacity of the lung for carbon monoxide; IPF: idiopathic pulmonary fibrosis. ${ }^{\#}$ : hazard ratios are given per 10 percentage-points difference in \% predicted FVC and $D$ Lco. ๆ: analysis restricted to patients with pulmonary function test within 1 year of diagnosis. 
a)

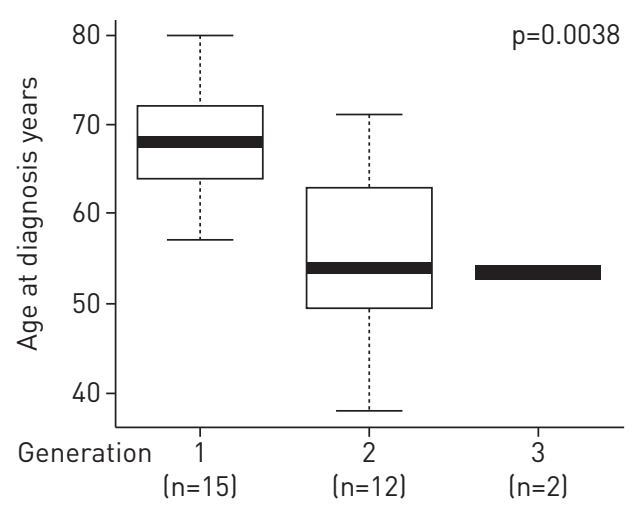

RTEL1

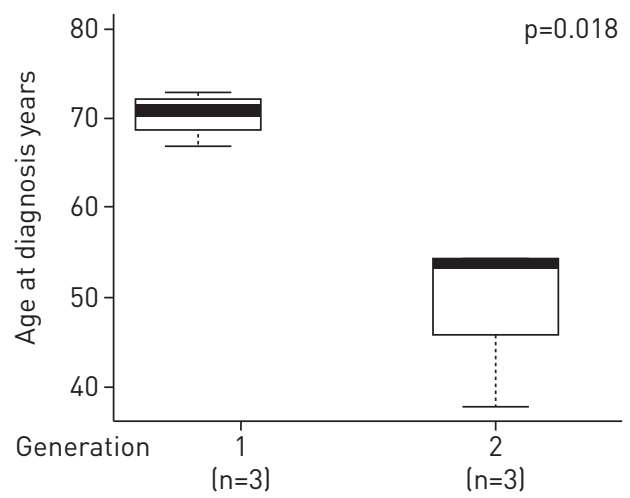

b)

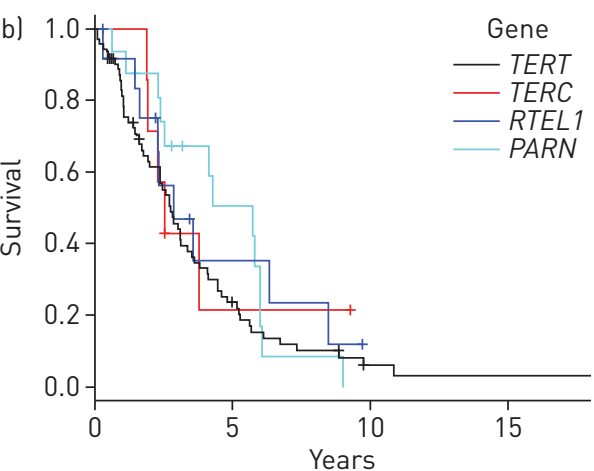

At risk $n$

TERT 71

TERC 7

RTEL 113

PARN 16

$\begin{array}{ccc}14 & 2 & 1 \\ 1 & 0 & 0 \\ 3 & 0 & 0 \\ 6 & 0 & 0\end{array}$

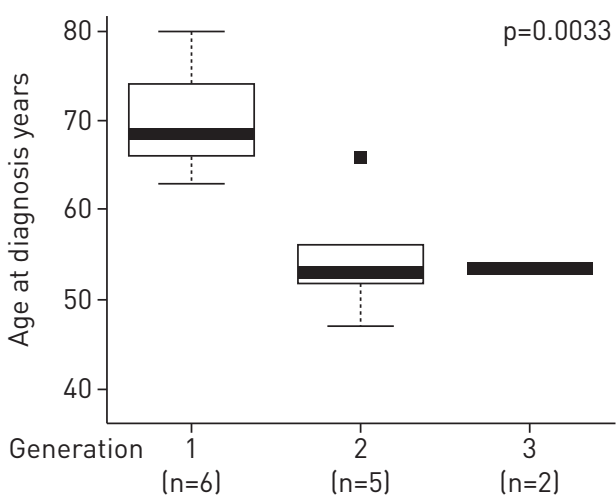

PARN
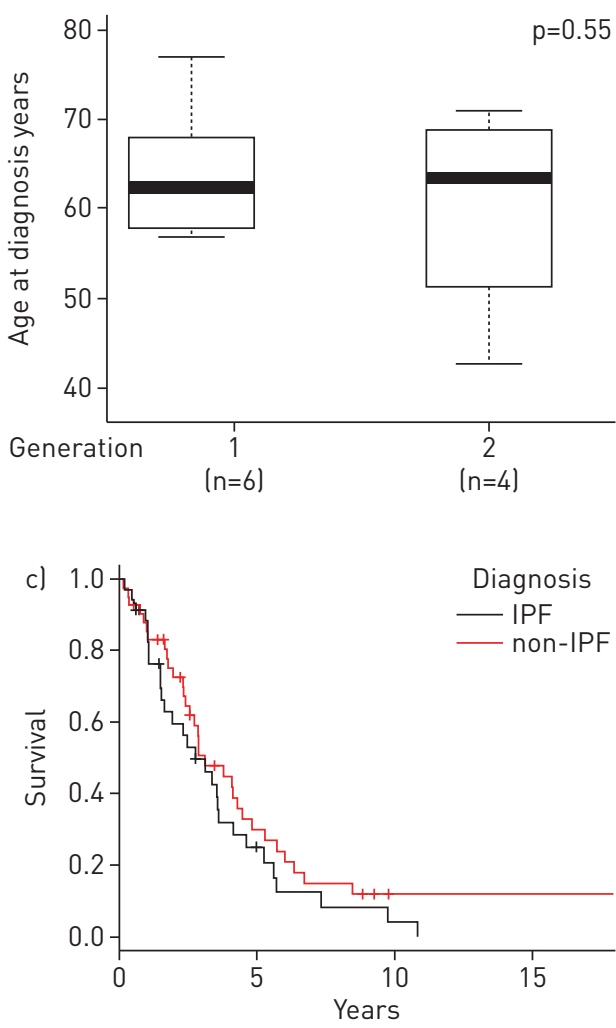

At risk $n$

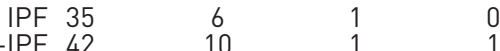

FIGURE 2 Clinical characteristics of interstitial lung disease (ILD) subjects with heterozygous TERT, TERC, RTEL 1 and PARN mutations. a) Evidence of genetic anticipation with an earlier age of ILD diagnosis for patients in subsequent generations of nine kindreds, including four with TERT mutations, two with RTEL1 mutations and three with PARN mutations. The number of individuals included in the analysis is listed on the $x$-axis. b) Transplant-free survival of ILD patients with TERT, TERC, RTEL1 and PARN mutations as depicted in a Kaplan-Meier survival plot. There is no significant difference between survival characteristics across different gene groups. c) Transplant-free survival of patients with TERT, TERC, RTEL1 and PARN mutations and a diagnosis of idiopathic pulmonary fibrosis (IPF) or a non-IPF diagnosis as depicted in a Kaplan-Meier survival plot. There was no significant difference between survival characteristics across the two diagnosis groups.

factor as we find that the subgroup of patients with pleuroparenchymal fibroelastosis is predominantly female $(88 \%)$. Other factors may include the duration and extent of fibrogenic environmental exposures as well as epigenetic or stochastic effects of telomere shortening in the certain lung cells. For example, there is evidence of airway-centric fibrosis and air trapping in individuals diagnosed with chronic hypersensitivity 
pneumonitis; in these cases, the inhaled fibrogenic environmental agent may have led to more rounds of cell division and more extreme telomere shortening of lung airway epithelia surrounding the small airways.

However, the one consistent phenotype seen across all genetic mutations is progressive deterioration. The mean rate of PFT decline seen in these monogenic ILD patients is more rapid than what is typically seen. We find a mean change in FVC of $-300 \mathrm{~mL} \cdot \mathrm{year}^{-1},-7.4 \mathrm{FVC} \%$ predicted per year and -5.8 DLCO \% predicted per year for subjects with heterozygous telomere-related gene mutations. In comparison, patients in the placebo arm of multiple IPF clinical trials demonstrated a median rate of FVC decline of $130-210 \mathrm{~mL} \cdot \mathrm{year}^{-1}$ [34] and subjects in the placebo arm of the Lung Scleroderma Study had a rate of fall of $2.6 \pm 0.9$ FVC \% predicted per year and 3.5 \pm 1.0 DLCO \% predicted per year [35]. Patients with these mutations represent an extreme phenotype, similar to those that have been classified with rapidly progressive disease [36, 37].

We found that there was no difference in survival of patients categorised by diagnosis, as those with a diagnosis of IPF have a median survival of 2.75 years (95\% CI 1.64-4.61) and those with a non-IPF diagnosis have a nearly equivalent survival of 3.11 years $(2.56-4.82)$. This finding is different from historic studies demonstrating a worse survival for IPF patients with a UIP histopathology [21] and suggests that the effect of the inherited telomere-related mutation is more predictive of accelerated progression than a particular clinical diagnosis or histopathological pattern. Given the lack of prognostic significance of histopathology and the morbidity associated with surgical lung biopsy, the risk and benefits of a surgical lung biopsy should be carefully considered for patients with a mutation in one of these telomere related genes. Increased vigilance and early referral for lung transplantation evaluation is needed, even for those with a non-IPF diagnosis.

Of the four gene mutation groups, we found the longest leukocyte telomere lengths in ILD subjects with PARN mutations which is consistent with prior reports [11]. The mean telomere lengths of patients in the different gene mutation groups fall in the following order: TERC $<T E R T<R T E L 1<P A R N$. The mean age of diagnosis for ILD patients in these different gene mutation groups was statistically significantly different and also follows this same order, with TERC mutation carriers diagnosed at the earliest age ( 51 years), followed by TERT mutation carriers (58 years), RTEL1 mutation carriers (60 years) and PARN mutation carriers (64 years). Similarly, there was a statistically significant higher prevalence of severe haematological comorbid conditions such as leukopenia, thrombocytopenia and aplastic anaemia/myelodysplastic syndrome in the TERC mutation group and a low prevalence of these haematological conditions in the RTEL1 and PARN mutation groups. Although not statistically significant, the mean time to death or transplant follows the same trend.

This study was an observational cohort and, as such, has been limited by ascertainment bias based upon the identification of an adult-onset pulmonary fibrosis phenotype. Patients received care at different locations and the practice patterns of their physicians varied widely. Despite our best efforts to provide a consistent multidisciplinary diagnostic approach by reviewing all primary clinical data, we were unable to establish a diagnosis for 38 patients because of missing or inaccessible chest CTs, surgical lung biopsies or lung explants. The study is also limited by a lack of comparison cohort controlling for regional and temporal clinical practices as well as genetic characteristics. Given the small numbers of patients with certain non-IPF diagnoses, such as unclassifiable pulmonary fibrosis and chronic hypersensitivity pneumonitis, we were unable to directly evaluate the rate of PFT progression or transplant-free survival for these individual groups. The large number of patients undergoing lung transplant may have influenced findings related to survival. However, sensitivity analyses censoring for transplant or treating it as a competing risk demonstrated qualitatively similar results (data not shown). Because of variable practices, we could not readily assess disease progression for IPF patients on FDA-approved medications or for patients with chronic hypersensitivity pneumonitis, connective tissue disease-associated ILD or IPAF on immunosuppression medications.

In summary, mutations in the telomere maintenance machinery genes TERT, TERC, RTEL1 and PARN lead to variable interstitial lung disease phenotypes and diagnoses. Although a specific ILD diagnosis may be important in terms of therapeutic options and prognosis for patients with sporadic disease, this study suggests that the effect of mutations in four different telomere-related genes predicts a similar pathogenic mechanism that manifests as progressive pulmonary fibrosis and reduced survival. Additional studies will be needed to determine the relative utility of telomere length and genetic mutations as biomarkers for predicting therapeutic treatments and outcomes in patients with familial pulmonary fibrosis.

\section{Acknowledgements}

The authors are grateful to the patients and the families for their participation in this research study and to Ashley Young (University of Texas Southwestern Medical Center, Dallas, TX, USA) for her technical expertise.

\section{References}

1 American Thoracic Society. Idiopathic pulmonary fibrosis: diagnosis and treatment. International consensus statement. American Thoracic Society (ATS), and the European Respiratory Society (ERS). Am J Respir Crit Care Med 2000; 161: 646-664. 
Raghu G, Collard HR, Egan JJ, et al. An official ATS/ERS/JRS/ALAT statement: idiopathic pulmonary fibrosis: evidence-based guidelines for diagnosis and management. Am J Respir Crit Care Med 2011; 183: 788-824.

3 Cronkhite JT, Xing C, Raghu G, et al. Telomere shortening in familial and sporadic pulmonary fibrosis. Am J Respir Crit Care Med 2008; 178: 729-737.

4 Alder JK, Chen JJ, Lancaster L, et al. Short telomeres are a risk factor for idiopathic pulmonary fibrosis. Proc Natl Acad Sci U S A 2008; 105: 13051-13056.

5 Stuart BD, Lee JS, Kozlitina J, et al. Effect of telomere length on survival in patients with idiopathic pulmonary fibrosis: an observational cohort study with independent validation. Lancet Respir Med 2014; 2: 557-565.

6 Armanios MY, Chen JJ, Cogan JD, et al. Telomerase mutations in families with idiopathic pulmonary fibrosis. N Engl J Med 2007; 356: 1317-1326.

7 Tsakiri KD, Cronkhite JT, Kuan PJ, et al. Adult-onset pulmonary fibrosis caused by mutations in telomerase. Proc Natl Acad Sci U S A 2007; 104: 7552-7557.

8 Diaz de Leon A, Cronkhite JT, Katzenstein AL, et al. Telomere lengths, pulmonary fibrosis and telomerase (TERT) mutations. PLoS One 2010; 5: e10680.

9 Kropski JA, Mitchell DB, Markin C, et al. A novel dyskerin (DKC1) mutation is associated with familial interstitial pneumonia. Chest 2014; 146: e1-e7.

10 Alder JK, Stanley SE, Wagner CL, et al. Exome sequencing identifies mutant TINF2 in a family with pulmonary fibrosis. Chest 2015; 147: 1361-1368.

11 Stuart BD, Choi J, Zaidi S, et al. Exome sequencing links mutations in PARN and RTEL1 with familial pulmonary fibrosis and telomere shortening. Nat Genet 2015; 47: 512-517.

12 Cogan JD, Kropski JA, Zhao M, et al. Rare variants in RTEL1 are associated with familial interstitial pneumonia. Am J Respir Crit Care Med 2015.

13 Kannengiesser C, Borie R, Menard C, et al. Heterozygous RTEL1 mutations are associated with familial pulmonary fibrosis. Eur Respir J 2015; 46: 474-485.

14 Moon DH, Segal M, Boyraz B, et al. Poly(A)-specific ribonuclease (PARN) mediates 3 '-end maturation of the telomerase RNA component. Nat Genet 2015; 47: 1482-1488.

15 Marrone A, Walne A, Tamary $\mathrm{H}$, et al. Telomerase reverse-transcriptase homozygous mutations in autosomal recessive dyskeratosis congenita and Hoyeraal-Hreidarsson syndrome. Blood 2007; 110: 4198-4205.

16 Walne AJ, Vulliamy T, Kirwan M, et al. Constitutional mutations in RTEL1 cause severe dyskeratosis congenita. Am J Hum Genet 2013; 92: 448-453.

17 Ballew BJ, Yeager M, Jacobs K, et al. Germline mutations of regulator of telomere elongation helicase 1, RTEL1, in Dyskeratosis congenita. Hum Genet 2013; 132: 473-480.

18 Deng Z, Glousker G, Molczan A, et al. Inherited mutations in the helicase RTEL1 cause telomere dysfunction and Hoyeraal-Hreidarsson syndrome. Proc Natl Acad Sci U S A 2013; 110: E3408-E3416.

19 Dhanraj S, Gunja SM, Deveau AP, et al. Bone marrow failure and developmental delay caused by mutations in poly(A)-specific ribonuclease (PARN). J Med Genet 2015; 52: 738-748.

20 Tummala H, Walne A, Collopy L, et al. Poly(A)-specific ribonuclease deficiency impacts telomere biology and causes dyskeratosis congenita. J Clin Invest 2015; 125: 2151-2160.

21 Bjoraker JA, Ryu JH, Edwin MK, et al. Prognostic significance of histopathologic subsets in idiopathic pulmonary fibrosis. Am J Respir Crit Care Med 1998; 157: 199-203.

22 Dai J, Cai H, Li H, et al. Association between telomere length and survival in patients with idiopathic pulmonary fibrosis. Respirology 2015; 20: 947-952.

23 Travis WD, Costabel U, Hansell DM, et al. An official American Thoracic Society/European Respiratory Society statement: update of the international multidisciplinary classification of the idiopathic interstitial pneumonias. Am J Respir Crit Care Med 2013; 188: 733-748.

24 Fischer A, Antoniou KM, Brown KK, et al. An official European Respiratory Society/American Thoracic Society research statement: interstitial pneumonia with autoimmune features. Eur Respir J 2015; 46: 976-987.

25 Armanios M. Telomerase and idiopathic pulmonary fibrosis. Mutat Res 2012; 730: 52-58.

26 Parry EM, Alder JK, Qi X, et al. Syndrome complex of bone marrow failure and pulmonary fibrosis predicts germline defects in telomerase. Blood 2011; 117: 5607-5611.

27 Jonassaint NL, Guo N, Califano JA, et al. The gastrointestinal manifestations of telomere-mediated disease. Aging Cell 2013; 12: 319-323.

28 Stanley SE, Chen JJ, Podlevsky JD, et al. Telomerase mutations in smokers with severe emphysema. J Clin Invest 2015; 125: 563-570.

29 Gorgy AI, Jonassaint NL, Stanley SE, et al. Hepatopulmonary syndrome is a frequent cause of dyspnea in the short telomere disorders. Chest 2015; 148: 1019-1026.

30 George G, Rosas IO, Cui Y, et al. Short telomeres, telomeropathy, and subclinical extrapulmonary organ damage in patients with interstitial lung disease. Chest 2015; 147: 1549-1557.

31 Tokman S, Singer JP, Devine MS, et al. Clinical outcomes of lung transplantation in patients with telomerase mutations. J Heart Lung Transplant 2015; 34: 1318-1324.

32 Steele MP, Speer MC, Loyd JE, et al. Clinical and pathologic features of familial interstitial pneumonia. Am J Respir Crit Care Med 2005; 172: 1146-1152.

33 Chung JH, Chawla A, Peljto AL, et al. CT scan findings of probable usual interstitial pneumonitis have a high predictive value for histologic usual interstitial pneumonitis. Chest 2015; 147: 450-459.

34 Ley B, Collard HR, King TE Jr. Clinical course and prediction of survival in idiopathic pulmonary fibrosis. Am J Respir Crit Care Med 2011; 183: 431-440.

35 Tashkin DP, Elashoff R, Clements PJ, et al. Cyclophosphamide versus placebo in scleroderma lung disease. N Engl J Med 2006; 354: 2655-2666.

36 Collard HR, King TE Jr, Bartelson BB, et al. Changes in clinical and physiologic variables predict survival in idiopathic pulmonary fibrosis. Am J Respir Crit Care Med 2003; 168: 538-542.

37 Zappala CJ, Latsi PI, Nicholson AG, et al. Marginal decline in forced vital capacity is associated with a poor outcome in idiopathic pulmonary fibrosis. Eur Respir J 2010; 35: 830-836. 